\title{
RUDIN-KEISLER POSETS OF COMPLETE BOOLEAN ALGEBRAS
}

\author{
PETER JIPSEN, ALEXANDER PINUS, HENRY ROSE
}

\begin{abstract}
The Rudin-Keisler ordering of ultrafilters is extended to complete Boolean algebras and characterised in terms of elementary embeddings of Boolean ultrapowers. The result is applied to show that the Rudin-Keisler poset of some atomless complete Boolean algebras is nontrivial.
\end{abstract}

\section{INTRODUCTION}

All concepts and notations not defined below can be found in [3].

Let $B$ be a Boolean algebra, and let $\mathbb{P}_{B}$ denote the set of all partitions of $B$ (i.e. maximal sets of pairwise disjoint elements). Note that $\mathbb{P}_{B}$ is ordered by the refinement relation: $\tau \leq \sigma$ if for all $x \in \tau$ there exists a $y \in \sigma$ such that $x \leq y$. Let $\hat{\sigma}=\bigcup\{\tau: \tau \leq \sigma\}$ be the set of nonzero elements of $B$ that are below some element of $\sigma$. Since $\sigma$ is a partition, each $x \in \hat{\sigma}$ is less than or equal to a unique $y \in \sigma$, so there is a natural map $j_{\sigma}$ from $\hat{\sigma}$ to $\sigma$ given by $j_{\sigma}(x)=y$. For a map $s: \sigma \rightarrow Y$ we define $\hat{s}=s \circ j_{\sigma}$, and occasionally we also abbreviate $\operatorname{dom} s$ by $s^{\mathrm{d}}$.

For $\sigma \in \mathbb{P}_{B}$ we let $\mathcal{P}(\sigma)$ be the powerset Boolean algebra over the set $\sigma$. If all joins of subsets of $\sigma$ exist in $B$ (e.g. if $B$ is $|\sigma|$-complete) then we identify $\mathcal{P}(\sigma)$ with the complete subalgebra of $B$ that is completely generated by $\sigma$.

For powerset Boolean algebras, the Rudin-Keisler ordering of ultrafilters is defined on $D \in \operatorname{Uf}(\mathcal{P}(X)), E \in \operatorname{Uf}(\mathcal{P}(Y))$ by $D \leq E$ if there exists a function $f: Y \rightarrow X$ such that

$$
\text { for all } S \in \mathcal{P}(X), \quad S \in D \text { implies } f^{-1}[S] \in E \text {. }
$$

We also write $D \leq_{f} E$ if $(*)$ holds. Note that this implication implies its converse, since $S \notin D$ implies $X \backslash S \in D$, hence $f^{-1}[X \backslash S]=Y \backslash f^{-1}[S] \in E$ and therefore $f^{-1}[S] \notin E$.

The duality between sets and powerset Boolean algebras implies the following equivalent definition: $D \leq E$ iff there exists a complete homomorphism $\alpha$ : $\mathcal{P}(X) \rightarrow \mathcal{P}(Y)$ such that $\alpha[D] \subseteq E$.

Date: June 7, 2004.

1991 Mathematics Subject Classification. 06E10, 03E55, 04A10.

Key words and phrases. Boolean algebras, partitions, Rudin-Keisler ordering.

The first and third author were supported by grants from the National Research Foundation of South Africa and the University of Cape Town Research Committee. The second author was supported by the Russian Foundation for Fundamental Researchers 99-01-00571. 
We wish to extend this ordering to complete (but not neccessarily atomic) Boolean algebras. Given a filter $D$ in a complete Boolean algebra $B$, and a partition $\sigma$ of $B$, we let $D_{\sigma}=D \cap \mathcal{P}(\sigma)$. Note that if $D$ is an ultrafilter of $B$, then $D_{\sigma}$ is an ultrafilter of $\mathcal{P}(\sigma)$. The idea of the definition below is to reduce the ordering of ultrafilters of $B$ and $C$, to the usual Rudin-Keisler ordering of the induced ultrafilters on complete and atomic subalgebras of $B$ and $C$. However, we need an additional concurrancy condition to ensure some nice properties of this extended ordering.

Definition 1.1. Let $B, C$ be complete Boolean algebras, $D \in \mathrm{Uf}(B)$ and $E \in$ $\mathrm{Uf}(C)$. We say that $D \leq E$ if there exists a map $g: \mathbb{P}_{B} \rightarrow \mathbb{P}_{C}$ and a family of maps $f_{\sigma}: g(\sigma) \rightarrow \sigma\left(\sigma \in \mathbb{P}_{B}\right)$ such that

(i) for all $S \subseteq \sigma, \quad \sum S \in D$ implies $\sum f_{\sigma}^{-1}[S] \in E$, (i.e. $D_{\sigma} \leq_{f_{\sigma}} E_{g(\sigma)}$ for all $\sigma \in \mathbb{P}_{B}$ ) and,

(ii) the family of $f_{\sigma}$ satisfies the following concurrancy condition

$$
\forall \tau, \sigma \in \mathbb{P}_{B}, \tau \leq \sigma \text { implies } \sum\left\{y \in g(\tau) \otimes g(\sigma): \hat{f}_{\tau}(y) \leq \hat{f}_{\sigma}(y)\right\} \in E .
$$

Here $\otimes$ is the meet operation in $\mathbb{P}_{B}$, i.e. $\sigma \otimes \tau$ is the greatest common refinement of $\sigma$ and $\tau$, given by $\{x y: x \in \sigma, y \in \tau\} \backslash\{0\}$. To make the connection with the previous version for powerset algebras, we have the following observation.

Proposition 1.2. Suppose $B, C$ and $D, E$ are as above, and $\alpha: B \rightarrow C$ is a complete homomorphism such that $\alpha[D] \subseteq E$. Then $D \leq E$.

Proof. Let $g: \mathbb{P}_{B} \rightarrow \mathbb{P}_{C}$ be defined by $g(\sigma)=\alpha[\sigma] \backslash\{0\}$. The completeness of $\alpha$ is needed to ensure that $\sum g(\sigma)=1$, and since $\alpha$ is meet-preserving, it is injective on families of disjoint elements that are not mapped to 0. Hence we can define an inverse $f_{\sigma}: g(\sigma) \rightarrow \sigma$ by $f_{\sigma}(y)=x$ iff $y=\alpha(x)$. Let $S \subseteq \sigma$, and suppose $\sum S \in D$. Then $\sum f_{\sigma}^{-1}[S]=\sum \alpha[S]=\alpha\left(\sum S\right) \in E$.

Finally, the concurrency condition holds in a somewhat stronger form: for $\tau \leq \sigma \in \mathbb{P}_{B}$, we have $g(\tau) \leq g(\sigma)$ and for all $y \in g(\tau), \hat{f}_{\tau}(y) \leq \hat{f}_{\sigma}(y)$.

The Rudin-Keisler ordering for complete Boolean algebras reduces to the usual ordering in case $B, C$ are powerset algebras. In one direction this follows immediately from the above proposition.

In the other direction, suppose $B=\mathcal{P}(X), C=\mathcal{P}(Y)$ and we are given a map $g: \mathbb{P}_{B} \rightarrow \mathbb{P}_{C}$, and maps $f_{\sigma}$ such that $D_{\sigma} \leq_{f_{\sigma}} E_{g(\sigma)}$. Consider the smallest partition $\sigma_{X}=\{\{x\}: x \in X\}$ in $\mathbb{P}_{B}$ and the corresponding smallest partition $\sigma_{Y} \in \mathbb{P}_{C}$. The required map $f: Y \rightarrow X$ is induced by the map $f_{\sigma_{X}} \circ j_{g\left(\sigma_{X}\right)}$ restricted to $\sigma_{Y}$, via the obvious isomorphism between a set and its collection of singleton subsets. Hence $D \leq E$ in the usual Rudin-Keisler order.

Problem 1.3. For which algebras does the converse of Proposition 1.2 hold? Note that it does hold for powerset algebras. 
The relation $\leq$ is a quasi-order on the class of all ultrafilters on complete Boolean algebras. We write $D \approx E$ if $D \leq E$ and $E \leq D$. When we restrict ourselves to a single algebra $B$, the partially ordered set of equivalence classes $\mathrm{Uf}(B) / \approx$ is denoted by $\operatorname{RK}(B)$.

\section{Characterisation By elementary EMBeddings}

For the RK-order on powerset Boolean algebras, Blass [1] proved the following characterisation theorem:

Theorem 2.1. Let $D \in \operatorname{Uf}(\mathcal{P}(X))$ and $E \in \operatorname{Uf}(\mathcal{P}(Y))$. The following are equivalent:

(i) $D \leq E$

(ii) for every structure $M$, there exists an elementary embedding from the ultrapower $M^{X} / D$ to $M^{Y} / E$.

Since we will generalise this result to the extended RK-order, we briefly recall the details of this fundamental result. Assuming $f: Y \rightarrow X$ is the function that establishes $D \leq E$, one can define a map $e: M^{X} / D \rightarrow M^{Y} / E$ by $e(s / D)=$ $(s \circ f) / E$, and this map is an elementary embedding since if $\phi$ is a formula in the language of $M$, and $s_{1}, \ldots, s_{n} \in M^{X}$ then

$$
\begin{aligned}
& \quad M^{X} / D \models \phi\left[s_{1} / D, \ldots, s_{n} / D\right] \\
& \text { iff }\left\{x \in X: M \models \phi\left[s_{1}(x), \ldots, s_{n}(x)\right]\right\} \in D \\
& \text { iff } f^{-1}\left[\left\{x \in X: M \models \phi\left[s_{1}(x), \ldots, s_{n}(x)\right]\right\}\right] \in E \\
& \text { iff }\left\{y \in Y: M \models \phi\left[s_{1}(f(y)), \ldots, s_{n}(f(y))\right]\right\} \in E \\
& \text { iff } M^{Y} / E \models \phi\left[e\left(s_{1} / D\right), \ldots, e\left(s_{n} / D\right)\right] .
\end{aligned}
$$

The converse requires the following definition:

Definition 2.2. For any set $A$, we let $\bar{A}$ be the complete structure on $A$, defined as the model in which every relation $R$ is the interpretation of some relation symbol, say $\bar{R}$, and and every function $f$ is the interpretation of some function symbol, say $\bar{f}$, respectively.

Now, given an elementary embedding $e$ from $\bar{X}^{X}$ to $\bar{X}^{Y}$, the map $f$ is obtained by choosing any representative of $e\left(i d_{X} / D\right)$, since for any $S \subseteq X$

$$
\begin{aligned}
& \quad S \in D \\
& \text { iff }\left\{x \in X: \bar{X} \models \bar{S}\left[i d_{X}(x)\right]\right\} \in D \\
& \text { iff } \bar{X}^{X} / D \models \bar{S}\left[i d_{X} / D\right] \\
& \text { iff } \bar{X}^{Y} / E \models \bar{S}\left[e\left(i d_{X} / D\right)\right] \\
& \text { iff }\{y \in Y: \bar{X} \models \bar{S}[f(y)]\} \in E \\
& \text { iff } f^{-1}[S] \in E .
\end{aligned}
$$


In order to generalise this result to the extended RK-order, we replace the ultrapowers above by Boolean ultrapowers. Recall that the (unbounded) Boolean power $M[B]$ of a model $M$ over a complete Boolean algebra $B$ can be constructed as a direct limit of powers $M^{\sigma}$, where $\sigma \in \mathbb{P}_{B}$ (see e.g. [5]). If $B$ is a powerset algebra $\mathcal{P}(X)$, this construction reduces to the ordinary power $M^{X}$. Similarly, for any ultrafilter $D$ of $B$, the Boolean ultrapower $M[B] / D$ is (isomorphic to) a direct limit of ultrapowers $M^{\sigma} / D_{\sigma}$, and when $B=\mathcal{P}(X)$, then $M[B] / D \cong M^{X} / D$. We include some of the details here, since they are relevant to the results of this section.

Definition 2.3. Let $M$ be a structure for some language $L$, and let $B$ be a complete Boolean algebra, with $D$ a filter in $B$. The structure $M[B] / D$ has as universe the set $\left(\bigcup_{\rho \in \mathbb{P}_{B}} M^{\rho}\right) / \theta_{D}$, where $\theta_{D}$ is the equivalence relation defined by

$$
s \theta_{D} t \quad \text { iff } \quad \sum\left\{x \in s^{\mathrm{d}} \otimes t^{\mathrm{d}}: \hat{s}(x)=\hat{t}(x)\right\} \in D
$$

Given an $n$-ary relation $R$ on $M$, and $s_{1} / D \ldots s_{n} / D \in M[B] / D$, we have

$$
\begin{gathered}
M[B] / D \models R\left[s_{1} / D \ldots s_{n} / D\right] \quad \text { iff } \\
\sum\left\{x \in s_{1}^{\mathrm{d}} \otimes \cdots \otimes s_{n}^{\mathrm{d}}: M \models R\left[\hat{s}_{1}(x) \ldots \hat{s}_{n}(x)\right]\right\} \in D .
\end{gathered}
$$

Thus $M[B] / D$ is also a structure of the language $L$, usually called the (unbounded) reduced Boolean power of $M$ (with respect to $B, D$ ). If we take $D$ to be the trivial filter $\{1\}$, we get the unbounded Boolean power $M[B]$, and if we take $D$ to be an ultrafilter, we get a Boolean ultrapower.

By an easy induction on the structure of formulas, it follows that if $D$ is an ultrafilter then (1) and (2) remain equivalent when $R$ is replaced by any formula.

Theorem 2.4. Let $B, C$ be complete Boolean algebras, $D \in \operatorname{Uf}(B)$ and $E \in$ $\mathrm{Uf}(C)$. The following are equivalent:

(i) $D \leq E$,

(ii) for any model $M$, there is an elementary embedding of $M[B] / D$ into $M[C] / E$,

(iii) there is an elementary embedding of $\bar{B}[B] / D$ into $\bar{B}[C] / E$.

Proof. Obviously (ii) implies (iii).

Assume (i) holds, and let $g$ and $f_{\sigma}$ be the associated maps for this inequality. Define $e: M[B] / D \rightarrow M[C] / E$ by $e(s / D)=\left(s \circ f_{s^{\mathrm{d}}}\right) / E$. It suffices to check that this map is elementary: Let $\phi\left(x_{1}, \ldots, x_{n}\right)$ be any formula in the language of $M$, 
and $s_{1} / D, \ldots, s_{n} / D \in M[B] / D$. Then

$$
\begin{aligned}
& \quad M[B] / D \models \phi\left[s_{1} / D, \ldots, s_{n} / D\right] \\
& \text { iff } \sum\left\{x \in s_{1}^{\mathrm{d}} \otimes \cdots \otimes s_{n}^{\mathrm{d}}: M \models \phi\left[\hat{s}_{1}(x), \ldots, \hat{s}_{n}(x)\right]\right\} \in D \\
& \text { iff } \sum f_{\tau}^{-1}\left[\left\{x \in \tau: M \models \phi\left[\hat{s}_{1}(x), \ldots, \hat{s}_{n}(x)\right]\right\}\right] \in E, \text { where } \tau=s_{1}^{\mathrm{d}} \otimes \cdots \otimes s_{n}^{\mathrm{d}} \\
& \text { iff } \sum\left\{y \in g(\tau): M \models \phi\left[\hat{s}_{1}\left(f_{\tau}(y)\right), \ldots, \hat{s}_{n}\left(f_{\tau}(y)\right)\right]\right\} \in E \\
& \text { iff } \sum\left\{y \in g\left(s_{1}^{\mathrm{d}}\right) \otimes \cdots \otimes g\left(s_{n}^{\mathrm{d}}\right): M \models \phi\left[s_{1}\left(\hat{f}_{s_{1}^{\mathrm{d}}}(y)\right), \ldots, s_{n}\left(\hat{f}_{s_{n}^{\mathrm{d}}}(y)\right)\right]\right\} \in E \\
& \text { iff } M[C] / E \models \phi\left[e\left(s_{1} / D\right), \ldots, e\left(s_{n} / D\right)\right]
\end{aligned}
$$

where the second last "iff" is justified by the concurrancy condition on the $f_{\sigma}$ : Since $\tau \leq s_{i}^{\mathrm{d}}$, it follows by concurrancy that

$$
\sum\left\{y \in g(\tau) \otimes g\left(s_{i}^{\mathrm{d}}\right): \hat{f}_{\tau}(y) \leq \hat{f}_{s_{i}^{\mathrm{d}}}(y)\right\} \in E
$$

for each $i=1, \ldots, n$, hence

$$
\sum\left\{y \in g(\tau) \otimes g\left(s_{1}^{\mathrm{d}}\right) \otimes \cdots \otimes g\left(s_{n}^{\mathrm{d}}\right): \hat{s}_{i}\left(\hat{f}_{\tau}(y)\right)=s_{i}\left(\hat{f}_{s_{i}^{\mathrm{d}}}(y)\right) \text { for all } i\right\} \in E .
$$

Now assume (iii) holds, and let $e$ be the given elementary embedding. Consider the identity map $\operatorname{id}_{\sigma}: \sigma \rightarrow \sigma \subseteq B$, with the codomain extended to the set $B$. Then $\operatorname{id}_{\sigma} / D$ is in $\bar{B}[B] / D$, so $e\left(\operatorname{id}_{\sigma} / D\right)$ is an equivalence class in $\bar{B}[C] / E$. For each $\sigma \in \mathbb{P}_{B}$, choose $f_{\sigma} \in e\left(\operatorname{id}_{\sigma} / D\right)$, and let $g(\sigma)=\operatorname{dom} f_{\sigma}$. We first argue that although $f_{\sigma}$ maps into $\bar{B}$, we can assume that it's range is entirely within $\sigma$ : Let $\bar{\sigma}$ be the relation symbol of $\bar{B}$ such that $\bar{B} \models \bar{\sigma}[x]$ iff $x \in \sigma$. Since $\sum\{x \in \sigma: \bar{B} \models$ $\left.\bar{\sigma}\left(\operatorname{id}_{\sigma}(x)\right)\right\}=1 \in D$, we have that $\bar{B}[B] \models \bar{\sigma}\left[\operatorname{id}_{\sigma} / D\right]$, hence $\bar{B}[C] \models \bar{\sigma}\left[e\left(\operatorname{id}_{\sigma} / D\right)\right]$. But this means that $\sum\left\{y \in g(\sigma): \bar{B}=\bar{\sigma}\left(f_{\sigma}(y)\right)\right\}=c \in E$. Therefore $f_{\sigma}(y) \in \sigma$ whenever $y \leq c$. Choose any fixed $b \in \sigma$ and define $f_{\sigma}^{\prime}: g(\sigma) \rightarrow \sigma$ by

$$
f_{\sigma}^{\prime}(y)= \begin{cases}f_{\sigma}(y) & \text { if } y \leq c \\ b & \text { otherwise }\end{cases}
$$

then $f_{\sigma}^{\prime} / E=f_{\sigma} / E$, so we can replace $f_{\sigma}$ by $f_{\sigma}^{\prime}$. 
Next we show that for all $S \subseteq \sigma, \sum S \in D$ iff $\sum f_{\sigma}^{-1}[S] \in E$. Let $\bar{S}$ be the relation symbol of $\bar{B}$ such that $\overline{\bar{B}} \models \bar{S}[x]$ iff $x \in S$. Then

$$
\begin{aligned}
& \quad \sum S \in D \\
& \text { iff } \sum\left\{x \in \sigma: \bar{B} \models \bar{S}\left[\operatorname{id}_{\sigma}(x)\right]\right\} \in D \\
& \text { iff } \bar{B}[B] / D \models \bar{S}\left[\operatorname{id}_{\sigma} / D\right] \\
& \text { iff } \bar{B}[C] / E \models \bar{S}\left[e\left(\operatorname{id}_{\sigma} / D\right)\right] \\
& \text { iff } \bar{B}[C] / E \models \bar{S}\left[f_{\sigma} / E\right] \\
& \text { iff } \sum\left\{y \in g(\sigma): \bar{B} \models \bar{S}\left[f_{\sigma}(y)\right]\right\} \in E \\
& \text { iff } \sum f^{-1}[\{x \in \sigma: \bar{B} \models \bar{S}[x]\}] \in E \\
& \text { iff } \sum f^{-1}[S] \in E .
\end{aligned}
$$

Finally we prove the concurrancy condition: Let $\tau \leq \sigma$, and let $\bar{R}$ be a relation symbol for the graph of $j_{\sigma} \uparrow_{\tau}$, i.e., $\bar{B} \models \bar{R}[b, c]$ iff $\bar{b} \in \tau, c \in \sigma$ and $b \leq c$. Then $\bar{B}[B] / D=\bar{R}\left[\operatorname{id}_{\tau} / D, \mathrm{id}_{\sigma} / D\right]$ since $\sum\left\{x \in \tau: \bar{B}=R\left[\operatorname{id}_{\tau}(x), \hat{\mathrm{id}}_{\sigma}(x)\right]\right\}=1$. Therefore $\bar{B}[C] / E \models \bar{R}\left[f_{\tau} / E, f_{\sigma} / E\right]$, which means that $\sum\{y \in g(\tau) \otimes g(\sigma): \bar{B} \models$ $\left.\bar{R}\left[\hat{f}_{\tau}(y), \hat{f}_{\sigma}(y)\right]\right\} \in E$. This is equivalent to the concurrancy condition.

Remark 2.5. In the definition of $D \leq E$, it suffices to consider partitions from a dense subsemilattice $S$ of $\mathbb{P}_{B}$. This follows from the characterisation theorem above since if $f \in B^{\sigma}$ for some $\sigma \in \mathbb{P}_{B}$, then there exists $\tau \in S$ with $\tau \leq \sigma$, and we may replace $f$ by $\hat{f} \uparrow_{\tau}$.

Example 2.6. Let $A=\prod_{i \in I} B_{i}$ be a product of complete Boolean algebras. Recall that each factor $B_{i}$ is isomorphically embedded into the relative subalgebra $A\left\lceil e_{i}\right.$, where $e_{i}$ is the $I$-tuple for which $e_{i}(i)=1_{B_{i}}$ and $e_{i}(j)$ is $0_{B_{j}}$ in all other coordinates $j \neq i$. We denote this relative embedding of $B_{i}$ into $A$ by $\gamma_{i}$. Observe that $\pi_{i} \circ \gamma_{i}$ is the identity function on $B_{i}$, and although $\gamma_{i}$ is not a homomorphism, it does preserve all existing joins and meets.

For a family of partitions $\sigma_{i} \in \mathbb{P}_{B_{i}}(i \in I)$ we define the partition product $\mathbb{X}_{i \in I} \sigma_{i}$ to be $\bigcup_{i \in I} \gamma_{i}\left[\sigma_{i}\right]$. This is easily seen to be a partition of $A$, and the set of all partition products forms a dense subsemilattice of $\mathbb{P}_{A}$.

Recall from [3] the definition of a relative subalgebra $B\lceil u$ of a Boolean algebra $B$ with $u \in B$. If $D \in \operatorname{Uf}(B)$ and $u \in D$, we let $D u=\{x \cdot u: x \in D\}$. Note that $D u$ is an ultrafilter in $B\lceil u$. With the characterisation theorem at hand, we get the following result.

Proposition 2.7. Let $B, C$ be complete Boolean algebras, and $D \in \operatorname{Uf}(B), E \in$ $\mathrm{Uf}(C)$. The following are equivalent:

(i) $D \leq E$, 
(ii) there exist $u \in D$ and $v \in E$ such that $D u \leq E v$,

(iii) for some $u \in D$ and some complete subalgebra $C^{\prime}$ of $C$, we have $D u \leq$ $C^{\prime} \cap E$.

Proof. (i) implies (ii), and (i) implies (iii) follow immediately if we take $u=$ $1_{B}, v=1_{C}$ and $C^{\prime}=C$. To prove (ii) implies (i), we observe that for any structure $M, M[B] / D \cong M[B\lceil u] / D u$, and by the preceeding theorem, the latter is elementarily embedded in $M[C\lceil v] / E v \cong M[C] / E$. Another application of the same theorem gives (i).

The implication from (iii) to (i) is proved similarly, using the additional fact that $M\left[C^{\prime}\right] /\left(C^{\prime} \cap E\right)$ is elementarily embedded in $M[C] / E$.

\section{Extending RK-POSETS}

In this section we look at conditions under which the RK-poset of one Boolean algebra is embedded in the RK-poset of another.

\subsection{Relative subalgebras.}

Lemma 3.1. Let $B$ be a Boolean algebra and $C=B\lceil a$ a relative subalgebra of $B$. If $D$ is an ultrafilter of $C$ then $\bar{D}=\{x \in B: x \geq y$ for some $y \in D\}$ is an ultrafilter of $B$.

Proof. By definition, $\bar{D}$ is up-closed, and since $D$ is meet-closed, the same holds true for $\bar{D}$. Therefore $\bar{D}$ is a filter. Given $x \in B$, we have $x \cdot a \in C$, hence $x \cdot a \in D$ or $-{ }^{a}(x \cdot a) \in D$. Since $-{ }^{a}(x \cdot a)=-x \cdot a$, we either have $x \in \bar{D}$ or $-x \in \bar{D}$, as required.

Corollary 3.2. If $C$ is isomorphic to a relative subalgebra of $B$, then $\operatorname{RK}(C)$ is embeddable into $\operatorname{RK}(B)$.

Proof. We can assume that $C=B\lceil a$ for some $a \in B$. Let $D, E \in \operatorname{Uf}(C)$. Then $D=\bar{D} a$ and $E=\bar{E} a$, so if $D \leq E$, then $\bar{D} \leq \bar{E}$ follows from Proposition 2.7(ii) $\Rightarrow$ (i).

Conversely, $\bar{D} \leq \bar{E}$ implies $D \leq E$ since relativization preserves the comparability of ultrafilters.

3.2. Powers of complete Boolean algebras. For a set $J$ and a complete Boolean algebra $B$, consider the direct power $B^{J}$. For ultrafilters $D$ in $B$, and $H$ in $\mathcal{P}(J)$, we define $D_{H}=\left\{s \in B^{J}: s^{-1}[D] \in H\right\}$.

If $B$ is a powerset algebra, say $\mathcal{P}(I)$, then $B^{J}$ is isomorphic to $\mathcal{P}(I \times J)$ and $D_{H}$ is isomorphic to the product ultrafilter $D \times H$ (as defined in [2]). It is straightforward to check that $D_{H}$ is an ultrafilter in this more general setting.

Lemma 3.3. Suppose $B$ is a complete Boolean algebra, $D \in \mathrm{Uf}(B), F \in$ $\mathrm{Uf}(\mathcal{P}(I))$ and $H \in \mathrm{Uf}(\mathcal{P}(J))$. If $F \leq H$ then $D_{F} \leq D_{H}$. 
Proof. Since $F$ and $H$ are ultrafilters in powerset algebras, we can use the original definition of the RK-order. Assume $F \leq H$, and let $h$ be the function from $J$ to $I$ such that for all $S \subseteq I, S \in F$ implies $h^{-1}[S] \in H$. To show that $D_{F} \leq D_{H}$, it suffices by Proposition 1.2 to define a complete homomorphism $\alpha: B^{I} \rightarrow B^{J}$ such that $\alpha\left[D_{F}\right] \subseteq D_{H}$. Given $s \in B^{I}$, we let $\alpha(s)=s \circ h$. Since the operations in $B^{I}$ are defined pointwise, this is a complete homomorphism, and for $s \in D_{F}$ we have $s^{-1}[D] \in F$, hence $(s \circ h)^{-1}[D]=h^{-1}\left[s^{-1}[D]\right] \in H$.

The reverse implication requires a bit more work and an additional assumption. A filter $D$ is said to be $\kappa$-complete if for any set $S \subset D$ with $|S|<\kappa$ we have $\prod S \in D$. For an ultrafilter in a complete Boolean algebra $B$, this is equivalent to the condition that for any $\sigma \in \mathbb{P}_{B}$ with $|\sigma|<\kappa$ we have $D \cap \sigma \neq \emptyset$ (see e.g. [4] 0.9).

Lemma 3.4. Suppose $B$ is a complete Boolean algebra, $D \in \mathrm{Uf}(B), F \in$ $\mathrm{Uf}(\mathcal{P}(I))$ and $H \in \mathrm{Uf}(\mathcal{P}(J))$. If $D$ is $|I|^{+}$-complete then $D_{F} \leq D_{H}$ implies $F \leq H$.

Proof. Suppose $D_{F} \leq D_{H}$. Then there exists a map $g: \mathbb{P}_{B^{I}} \rightarrow \mathbb{P}_{B^{J}}$ and maps $h_{\gamma}: g(\gamma) \rightarrow \gamma \in \mathbb{P}_{B^{I}}$ such that for all $S \subseteq \gamma, \sum S \in D_{F}$ implies $\sum h_{\gamma}^{-1}[S] \in D_{H}$.

Consider the partition $\sigma_{I}=\left\{\chi_{\{i\}} \in B^{I}: i \in I\right\}$ and the corresponding partition $\sigma_{J} \in \mathbb{P}_{B^{J}}$, where $\chi_{K}$ is the charateristic function of $K \subseteq I$ or $J$ respectively. Let $\alpha$ be the complete homomorphism from $\mathcal{P}\left(\sigma_{I}\right)$ to $\mathcal{P}\left(g\left(\sigma_{I}\right)\right)$ given by $\alpha\left(\sum S\right)=$ $\sum h_{\sigma_{I}}^{-1}[S]$.

To show that $F \leq H$, we need to define a map $h: J \rightarrow I$ such that $S \in F$ implies $h^{-1}[S] \in H$ for all $S \subseteq I$. Given $j \in J$ and $i \in I$, let $h(j)=i$ iff $\pi_{j}\left(\alpha\left(\chi_{\{i\}}\right)\right) \in D$. The map is well-defined for all $j \in J$ since we are assuming that $D$ is $|I|^{+}$-complete, so the partition $\pi_{j} \circ \alpha\left[\sigma_{I}\right] \backslash\{0\}$ intersects $D$, and since $D$ is a filter, this intersection is a singleton.

Let $S \in F$. This is equivalent to $\left\{i \in I: \chi_{S}(i) \in D\right\} \in F$, and hence to $\chi_{S} \in D_{F}$. It follows that $\alpha\left(\chi_{S}\right) \in D_{H}$ and therefore $\alpha\left(\chi_{S}\right)^{-1}[D] \in H$. The following equivalent statements show that $\alpha\left(\chi_{S}\right)^{-1}[D]=h^{-1}[S]$ :

$$
\begin{aligned}
& j \in \alpha\left(\chi_{S}\right)^{-1}[D] \\
& \text { iff } \\
& \alpha\left(\chi_{S}\right)(j) \in D \\
& \text { iff } \\
& \sum_{i \in S} \alpha\left(\chi_{\{i\}}\right)(j) \in D
\end{aligned}
$$


Theorem 3.5. Let $B$ be a complete Boolean algebra, and suppose there exists a $\kappa^{+}$-complete ultrafilter in $B$. Then the poset $\operatorname{RK}(\mathcal{P}(\lambda))$ is order embeddable into the poset $\operatorname{RK}\left(B^{\kappa}\right)$ for any $\lambda \leq \kappa$.

If $B$ is homogeneous and contains a partition of size $\kappa$ then $B^{\kappa} \cong B$. Hence if $B$ has a $\kappa^{+}$-complete ultrafilter then $\operatorname{RK}(\mathcal{P}(\kappa))$ is order embeddable into $\operatorname{RK}(B)$.

An example of such a boolean algebra $B$ is given by the collapsing algebra $\operatorname{Col}\left(\kappa^{+}, \lambda\right)$ if we assume that $\kappa^{+}$is strongly inaccessible and $\left|\operatorname{Col}\left(\kappa^{+}, \lambda\right)\right|$-almost compact (see [4] Theorem 3.6), or if we assume that $\kappa^{+}$is measurable.

Problem 3.6. Can the above theorem be proved in ZFC (i.e. without the large cardinal assumption about the existence of a $\kappa^{+}$-complete ultrafilter)?

\section{REFERENCES}

[1] A. Blass, Orderings of Ultrafilters, thesis, Harvard University, 1970.

[2] C. C. Chang and H. J. Keisler, "Model Theory", North-Holland, 1977.

[3] "Handbook of Boolean Algebras", ed. J. D. Monk and R. Bonnet, Vol 1-3, North-Holland, 1989.

[4] P. Jipsen and H. Rose, Partition complete Boolean algebras and almost compact cardinals, Mathematical Logic Quarterly, 45 (1998) 2, 241-255.

[5] P. Ouwehand and H. Rose, Filtral powers of structures, Journal of Symbolic Logic, Vol 63, No 4 (1998), 1239-1254.

Department of Mathematics, Vanderbilt University, Nashville, TN 37240, USA

Department of algebra and mathematical logic, Novosibirsk State Technical University, PR. K.MarXa 20, 630092, Novosibirsk, Russia

Department of Mathematics and Applied Mathematics, University of Cape Town, Rondebosch, 7701, South Africa

E-mail address: pjipsen@math.vanderbilt.edu

E-mail address: algebra@redhouse.nstu.nsk.su

E-mail address: rose@maths.uct.ac.za 